\title{
Estimate of economic impact of atmospheric radiation storm associated with solar energetic particle events on aircraft operations
}

\author{
Susumu Saito ${ }^{1 *} \mathbb{D}$, Navinda Kithmal Wickramasinghe ${ }^{1}$, Tatsuhiko Sato ${ }^{2}$ and Daikou Shiota ${ }^{3}$
}

\begin{abstract}
A solar energetic particle (SEP) event generates a shower of secondary generated particles in the Earth's atmosphere down to lower altitudes to cause an atmospheric radiation storm (ARS). The high-energy secondary particles cause additional radiation dose at altitudes where aircraft flies. The space weather information provided by the International Civil Aviation Organization (ICAO) designated space weather centers includes advisories on the solar radiation storm. The Warning System for AVlation Exposure to Solar energetic particle (WASAVIES), we can estimate the effective dose rate (EDR) along the flight path of the aircraft. However, it has not been well established how the operators of aircraft should react with the space weather advisories on the solar radiation storm. By using a flight trajectory generation algorithm and the global EDR distribution, the economic impacts of ARS associated with SEP events on aircraft operation, namely the flight path length, flight time, and fuel consumption, are estimated. The conditions of the peak of the ARS event on 20 January 2005 are used. The economic impacts for a flight route from New York, US to Tokyo, Japan, are estimated with constraints in flight routes to avoid the hazard of radiation and compared with those of the reference case without the ARS effects. The fuel consumption is shown to increase by 39-69 tons (33-58\%) for a twin-engine, wide-body jet passenger aircraft, when a constraint is imposed on the flight altitude only. When the constraints are set on the aircraft altitude and the latitude, the flight time and the fuel consumption are both increased by $2.2-2.8 \mathrm{~h}(17-20 \%)$ and $32-48$ tons (27-41\%), respectively. If the ARS event duration is limited for $3 \mathrm{~h}$, the increase in the fuel consumption is about 7.6-14 tons (6.4-12\%). This economic impact may be reduced, if the space weather nowcast and forecast for the ARS and an optimal flight trajectory generation algorithm are used together. Setting more flexible constraints on the flight route and generating optimal flight trajectories with minimal economic impacts by fully utilizing the global EDR distribution is the next step.
\end{abstract}

Keywords: Solar energetic particle event, Radiation, Impact on airplane operation

\section{Introduction}

A solar energetic particle (SEP) event is a high-energy particle stream from the Sun mainly consisting of energetic protons. The high-energy particles cannot directly penetrate into the Earth's atmosphere due to the Earth's magnetic field, but come into the Earth's atmosphere

\footnotetext{
*Correspondence: susaito@mpat.go.jp

1 Electronic Navigation Research Institute, National Institute of Maritime, Port and Aviation Technology, 7-42-23 Jindaiji-Higashi, Chofu, Tokyo 182-0012, Japan

Full list of author information is available at the end of the article
}

along the magnetic field in the high-latitude regions. Higher energy particles can penetrate to lower altitudes and lower latitudes to cause an atmospheric radiation storm. The high-energy particles collide with the atoms of the atmosphere to generate secondary particles by a cascading process. The generated high-energy secondary particles cause increased radiation dose for passengers and crew onboard aircraft flying at high altitudes in addition to the radiation caused by the galactic cosmic rays which normally exists. The additional dose by the 
ARS event is known to be sometimes so intense as to be a potential hazard to the passengers and crew onboard (Lantos and Fuller 2003; Mekhaldi et al. 2015).

The International Civil Aviation Organization (ICAO) has started a service from November 2019 to provide information on space weather conditions to support safe and efficient air navigation (ICAO 2018a). The provided space weather information includes advisories on solar radiation storms which will be issued when the radiation dose associated with the ARS events are likely to cross the defined thresholds.

The Warning System for AVIation Exposure to Solar energetic particle (WASAVIES) has been developed to nowcast the ARS associated with SEP events and global distribution of the dose rate at various altitudes (Kataoka et al. 2014; Sato et al. 2018). It is a physics-based SEP transport model with inputs of proton fluxes from Geostationary Operational Environmental Satellite (GOES) and count rates of several neutron monitors at the ground level to determine free parameters in the model. It has been used for the space weather information service provided by one of the ICAO space weather global center operated by Australia, Canada, France and Japan (ACFJ) (WASAVIES 2020). There are other systems that can provide nowcast of the dose rate in ARS events such as SiGLE (Lantos et al. 2003), AVIDOS (Latocha et al. 2009, 2015), NAIRAS (Mertens et al. 2010), and CARI-7/CARI-7A (Copeland 2014, 2017).

Because the space weather information has to be used by users, just providing information is not sufficient, but it is necessary to combine the space weather information with aviation operations in the real world. However, it has not been well established how the operators of aircraft should react to the space weather advisory on the solar radiation storms. Because safety is the first priority in civil aviation, the most conservative reaction would be to cancel flights, However, if there is quantitative information to allow the aviation operators to make decisions in response to the space weather advisory, there could be two kinds to reactions. One is for aircraft in flight, and the other is for aircraft before flight. For aircraft in flight, one of the possible ways is to lower the flight altitude (Mattiä et al. 2015). For aircraft before flight, whole flights could be planned to sufficiently reduce the radiation dose. However, the civil aviation is an economic activity, so economic impacts need to be minimized while keeping the safety at a required level. Therefore, it is necessary to be able to estimate the economic impacts on civil aviation when the additional radiation dose is expected to cause hazard and flight routes have to be planned to avoid the radiation dose.

In the field of the air traffic management, there are studies to find flight trajectories under a certain set of constraints. Wickramasinghe et al. (2014) has developed a method to generate flight trajectories by using an aircraft performance database and an aircraft three-dimensional dynamics model. They used the method to optimize flight paths and assess the operational performance of aircraft (Wickramasinghe et al. 2017a, b). By using a database of performance of existing aircraft, they can design an optimal flight path by setting various conditions such as aircraft type, aircraft weight, wind speed, and constraints such as the maximum flight altitude, and the maximum air speed. They can also derive the flight path information such as flight path length, flight time, and fuel consumption of the flight. By combining the ARS nowcasting system and the flight path optimization algorithm, it is possible to estimate how the flight path would be changed to avoid the hazard associated with the solar radiation storm.

The main objective of this study is to estimate the economic impact including increased fuel consumption and delay of aircraft arrival when the aircraft is rerouted to ensure safety of the passengers and crew members under ARS events. To estimate the upper limit of the impacts, extreme ARS condition is considered. This study develops methodology of economic impact estimation associated with ARS events and shows some examples to be a basis of further detailed studies.

\section{Methods}

\section{Selection of a representative flight route}

In this study, an flight route from New York John F. Kennedy International Airport (JFK; $40.6^{\circ} \mathrm{N}, 73.8^{\circ} \mathrm{W}$ ) to Tokyo Narita International Airport (NRT; $35.8^{\circ} \mathrm{N}$, $\left.140.4^{\circ} \mathrm{E}\right)$ is chosen as an example. This route is chosen among the flight routes departing from or arriving in Japan, because it transits high latitudes where SEP effects are most prominent. Figure 1 shows a real flight path of a flight JL003 on 31 January 2019 as recorded by Flight Radar 24 (FlightRadar24 2020), which is chosen as a typical flight on this route. The aircraft type of the flight is Boeing 777-300ER.

\section{ARS event selection and estimation of effective dose rate}

The ARS event which occurred associated with the SEP event on 20 January 2005 is chosen as a reference event, because it is the strongest event in the period when the data required by the WASAVIES to estimate the global distribution of the effective dose rate (EDR) are available. This event is also known as the GLE (Ground Level Enhancement)-69, which is the 69th event where the neutron enhancement is observed at the ground level. The radiation peaked at 0655 UT on 20 January 2005. We use the global EDR distribution at the peak of the event and assume that the condition lasts over the whole flight time. This conservative assumption does not come from the expected ARS event duration, but from a flight planning perspective. 
Operators would prefer a safe plan when a reliable prediction of the ARS event progress is not available in real-time operation. They would not try to make complicated flight path alternations during the flight as well. This assumption may also cover the possibility of even stronger ARS events than the one on 20 January 2005, which is the strongest event in the past 20 years. Figure 2a shows the estimated EDR at the flight level (FL) of 400 or the altitude of $40,000 \mathrm{ft}$ $(12.19 \mathrm{~km})$ where the aircraft cruises. Please note that the aircraft altitude is expressed in feet instead of meters or kilometers, because it is still commonly used in the field of aviation. As it can be seen, the EDR was significantly higher in the southern hemisphere, which could be due to the tilt angle of the interplanetary magnetic field (Sato et al. 2018). As flipped interplanetary magnetic field condition may occur, we flip the global EDR distribution axisymmetrically as shown in Fig. $2 b$ to use as the reference global EDR distribution to evaluate the effects on the flight in the northern hemisphere by applying the more severe condition in the southern hemisphere.

\section{Thresholds for effective dose by ARS}

To define alternative flight routes to avoid the radiation risk, criteria for acceptable radiation levels need to be defined. The International Commission on Radiological Protection (ICRP) recommends limiting the public exposure to be less than $1 \mathrm{mSv}$ per year (ICRP 2007). For a passenger on an aircraft flight for $10 \mathrm{~h}$, if the EDR was $100 \mu \mathrm{Svh}^{-1}$ during the whole flight, the total effective dose will reach the limit for one year in just one flight. Even though it would not be realistic that the ARS peak of $100 \mu \mathrm{Svh}^{-1}$ lasts for $10 \mathrm{~h}$, the EDR for passengers on aircraft, and especially for crew members who fly more frequently, should be much less than the $100 \mu \mathrm{Svh}^{-1}$. ICAO has defined the warning level of ARS associated with SEP events by the level of the possible EDR. There are two levels, moderate and severe, with corresponding thresholds of EDR of 30 and $80 \mu \mathrm{Svh}^{-1}$ at aircraft cruising altitudes, respectively (ICAO 2018b). The quiet time EDR at the cruising altitudes of aircraft at high-latitude region is about $10 \mu \mathrm{Svh}^{-1}$ (Sato 2015). In this study, we chose two thresholds to limit the maximum EDR on aircraft. One is $30 \mu \mathrm{Svh}^{-1}$ which corresponds to the moderate level of the ICAO space weather forecast, and the other is $10 \mu \mathrm{Svh}^{-1}$ which corresponds to the quiet time EDR as an extreme test case. The flight trajectories are calculated so that the EDR at the altitudes where the aircraft flies would not exceed the thresholds.

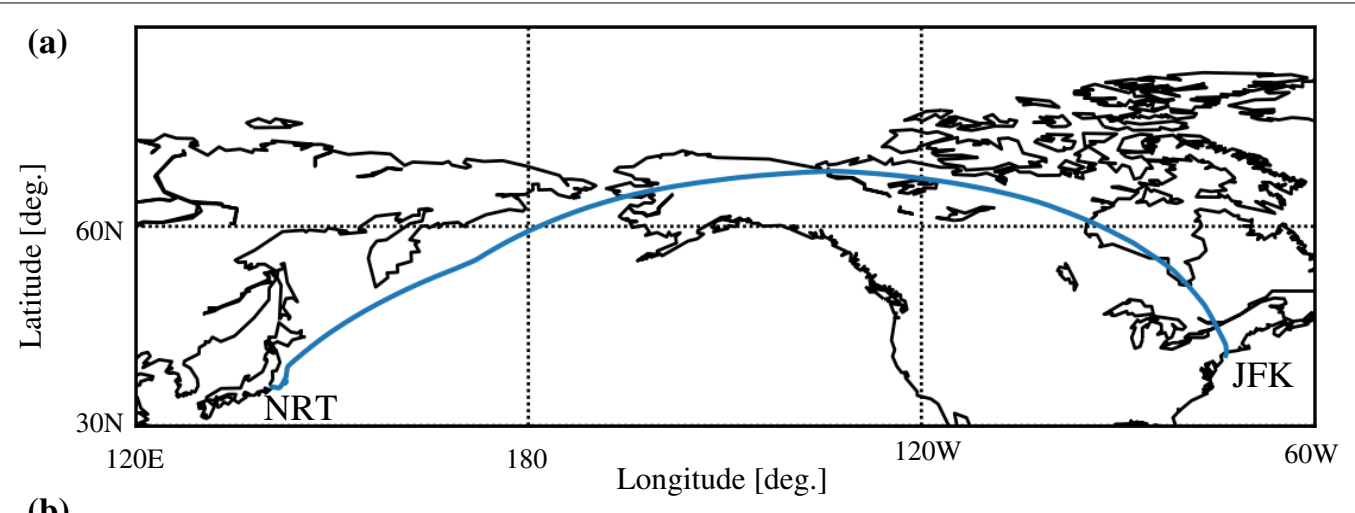

(b)

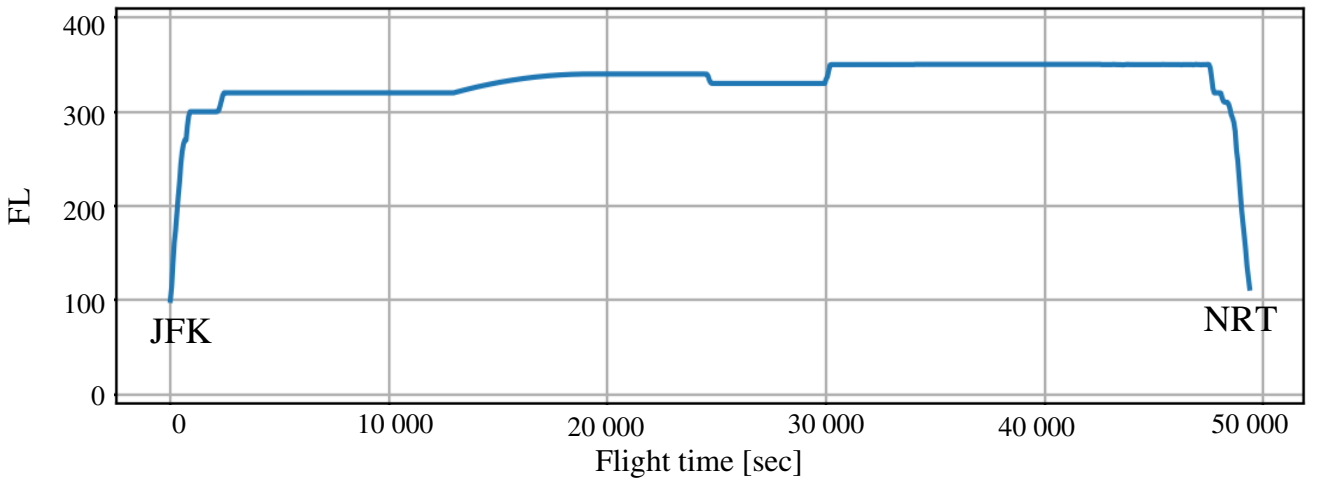

Fig. 1 a Lateral flight path from JFK to NRT (JL003) on 31 January 2020. b Flight altitudes in the unit of 100 feet (FL) from JFK to NRT (JL003) on 31 January 2020 


\section{Flight trajectory and parameter estimation}

Once the constraints on the EDR along the flight path have been defined, flight scenarios to satisfy the constraints are developed, as described in the next section. For each scenario, the flight path length, flight time, and fuel consumption are estimated. The estimation method assumes a scenario where the aircraft is required to follow the above-defined constraints while maintaining the original Mach speed (relative velocity to the acoustic velocity) profile. It is worthwhile noting that assumption of the same Mach number is reasonable when the flight altitudes are not very different from the original, but it may be unrealistic when the flight altitudes are very different from the original. The economic impacts on the operations are investigated by comparing the estimated flight path length, flight time and fuel consumption to the reference flight without constraint. Aircraft performance parameters are obtained from the Base of Aircraft Data (BADA) model developed by EUROCONTROL, which covers various types of aircraft (Poles 2009; Mouillet 2017). The conditions of the estimation to be supplied to the flight trajectory generation algorithm is as follows: the aircraft type is a twin-engine, wide-body jet passenger aircraft with the take-off weight of $320,915 \mathrm{~kg}$, which are a typical aircraft type and take-off weight used for longhaul flights. the wind speeds during the flight were taken from the Grid Point Value (GPV) model on the day of the flight (31 January 2019) provided by the Japan Meteorological Agency. The wind condition was a typical one in winter in the northern hemisphere. In addition, restricting conditions to avoid radiation by ARS are added in this study, which is described in the next section.

\section{Flight scenarios}

The flight path recorded by FlightRadar24 (2020) is used as the reference scenario. Figure 3 shows the flight altitudes and expected EDR along the flight path for the EDR distribution at the peak of the event. If the aircraft flew without constraint, it would have experienced the EDR up to $160 \mu \mathrm{Svh}^{-1}$. The lowest altitudes at which the EDR is 30 and $10 \mu \mathrm{Svh}^{-1}$ along the lateral flight path are 20,200 and $14,500 \mathrm{ft}$, respectively. The simplest but conservative way to protect aircraft passengers and crew members is to limit the aircraft altitude all through the flight. We set up two scenarios with a constraint on the aircraft altitude only. Corresponding to the two thresholds in EDR, 30 and $10 \mu \mathrm{Svh}^{-1}$, the aircraft altitudes are restricted to be lower than FL $200(6.10 \mathrm{~km})$ and 140 $(4.27 \mathrm{~km})$, respectively. It is not realistic to fly at such low altitudes as the fuel increase would be significant and there would be more risks to encounter severe meteorological conditions. Therefore, these scenarios should be regarded as test cases for extreme conditions.
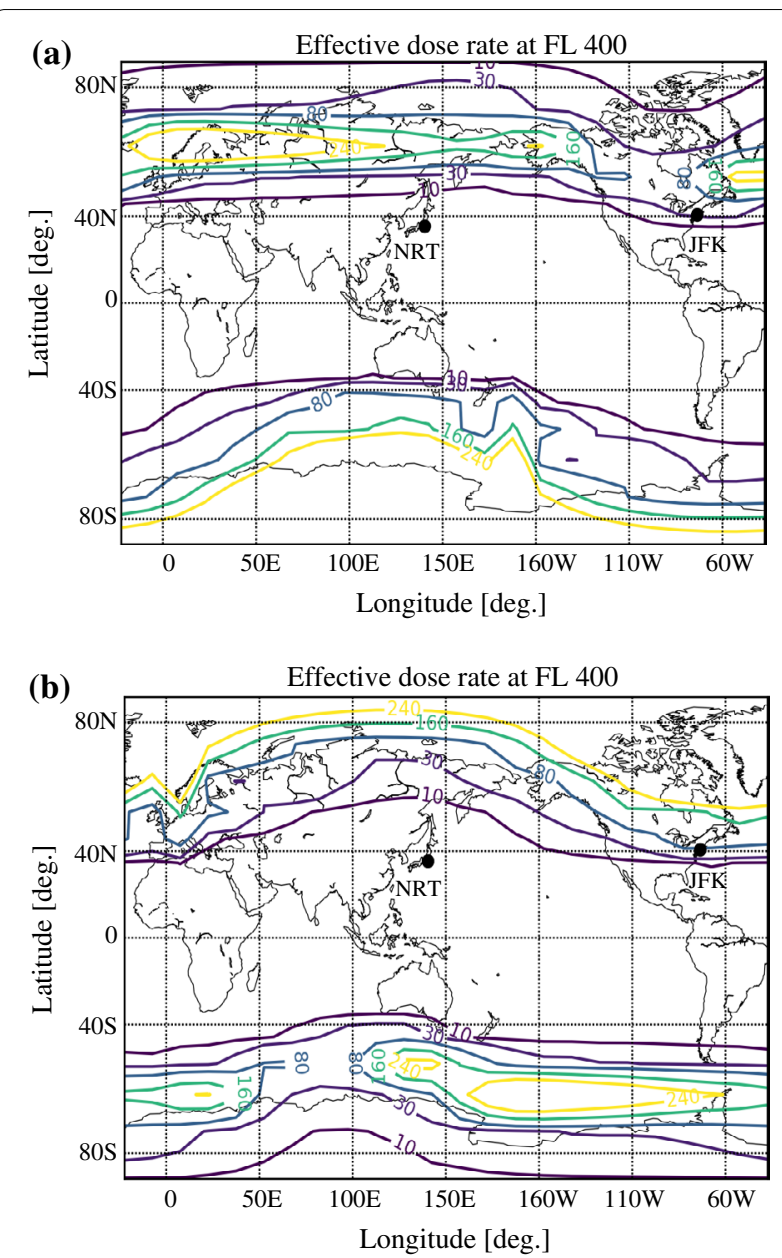

Fig. 2 a Estimated EDR in $\mu \mathrm{Sv}^{-1}$ at FL $400(12.19 \mathrm{~km})$ at 0655 UT on 20 January 2005. b Estimated EDR at an altitude of FL 400 (12.19 km) at 0655 UT on 20 January 2005, but with flipping the northern and southern hemispheres

However, these constraints may be too conservative, because the EDR would be lower at lower latitudes. By taking advantage of the latitudinal dependence of the EDR and by imposing the constraint on the latitude, the constraint on the aircraft altitude can be relaxed. As the fuel consumption would increase when the aircraft flies at lower altitudes, relaxing the constraint on the aircraft altitude may reduce the fuel consumption compared to the scenarios with the aircraft altitude constraint only. Figure $4 \mathrm{a}, \mathrm{b}$ shows the altitudes at which the EDR is 30 and $10 \mu \mathrm{Svh}^{-1}$, respectively. It can be seen that the altitudes of a constant EDR value are lower around JFK. For the EDR threshold of $10 \mu \mathrm{Svh}^{-1}$, for example, an aircraft cannot climb to FL 250 over JFK. Again to simplify the calculation, we choose just one set of the maximum allowed altitude and the maximum allowed latitude for each EDR thresholds. For the EDR threshold 
of $30 \mu \mathrm{Svh}^{-1}$, we set a constraint on the latitude as $44^{\circ} \mathrm{N}$ and relax the constraint in the altitude to FL 300 $(9.14 \mathrm{~km})$. The constraint on the latitude is set as $42^{\circ} \mathrm{N}$ for the EDR threshold of $10 \mu \mathrm{Svh}^{-1}$ with the constraint on the aircraft altitude of FL $300(7.62 \mathrm{~km})$. Obviously, the aircraft latitude has to be more limited than the $30 \mu \mathrm{Svh}^{-1}$ case. Table 1 summarizes the four restricted flight scenarios. Together with the reference scenario taken from the real trajectory and without constraint, the flight path length, flight time, and fuel consumption are calculated for in total five scenarios.

\section{Results and discussion}

Table 2 summarizes the results of the calculations for the five scenarios. Figure $5 \mathrm{a}, \mathrm{b}$ shows the lateral flight paths and the flight altitudes, respectively. When the constraint is imposed on the aircraft altitude only, the lateral paths are identical to that of the reference scenario. Therefore, the flight path lengths are the same for scenarios 1, 2 and 4, while the aircraft altitudes follow the maximum allowed altitudes. In scenarios 2 and 4, the flight times were shortened by $43-65 \mathrm{~min}$. This is because the true speed at lower altitudes is faster than at higher altitude, when the Mach number, which is the speed relative to the acoustic velocity, is maintained as assumed. However, flying at lower altitudes at faster speed result in the increased fuel consumption by 39-69 tons which corresponds to $33-58 \%$ of the reference scenario. As mentioned before, flying at very low altitude at the speed with the same Mach number as much higher altitudes may not be realistic. Therefore, this values of the increase in fuel consumption should be considered as values at the conservative limit. For scenarios 3 and 5, the flight paths follow the maximum allowed latitudes and altitudes. As a result, the flight path lengths were increased by about $1500 \mathrm{~km}$. The increased flight path lengths result in the increased flight time of about 2.2-2.8 $\mathrm{h}$ (17-20\% of the reference). The fuel consumption is also increased by $32-48$ tons (27-41\% of the reference). This increase in the fuel consumption is a combined effect of the increased flight path length and the increased air drag in flying at lower altitudes. However, the increases in the fuel consumption in scenarios 3 and 5 are less than those in scenarios 2 and 4.

As of February 2020, the price of the jet fuel is about 9000 JPY per barrel (159 l). The mass density of the Jet-A1 aviation fuel is $0.7753-0.8398 \mathrm{~kg} \mathrm{l}^{-1}$ (ASTM 2020). To be conservative, if we use the mass density of $0.78 \mathrm{~kg} \mathrm{l}^{-1}$, the price of the jet fuel is $72.6 \mathrm{JPY} \mathrm{kg}^{-1}$. For scenarios studied here, the increases in fuel cost is about 2.29-5.01 million JPY (or about 21-46 thousand USD) per flight. The increased costs associated with the delay of arrival, which we have avoided translating to economic cost, should be added. Although the assumptions in this analysis are quite conservative and constraints in calculation are simplified, these results can be interpreted that the economic impacts of an ARS event associated with the SEP event of

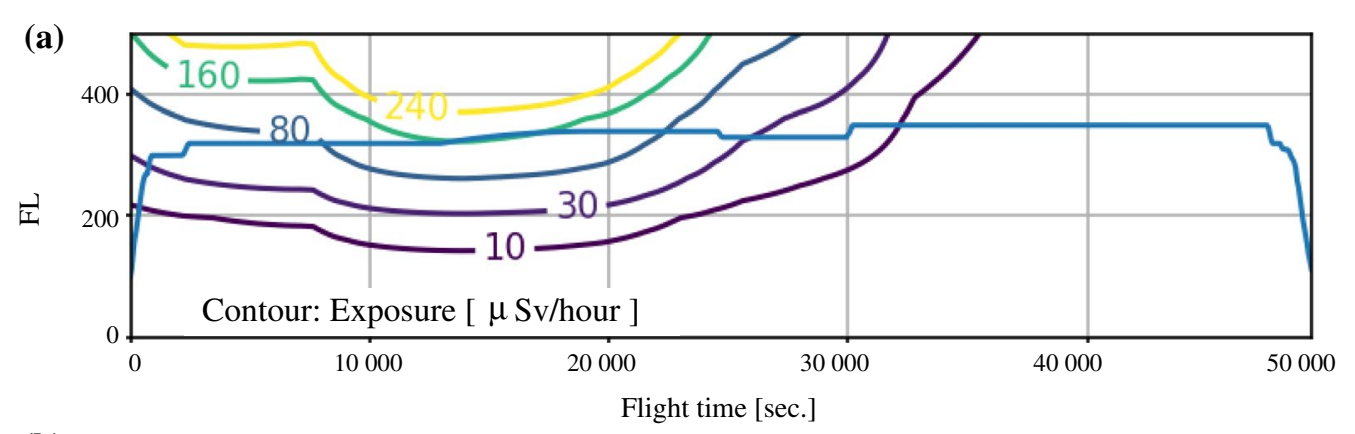

(b)

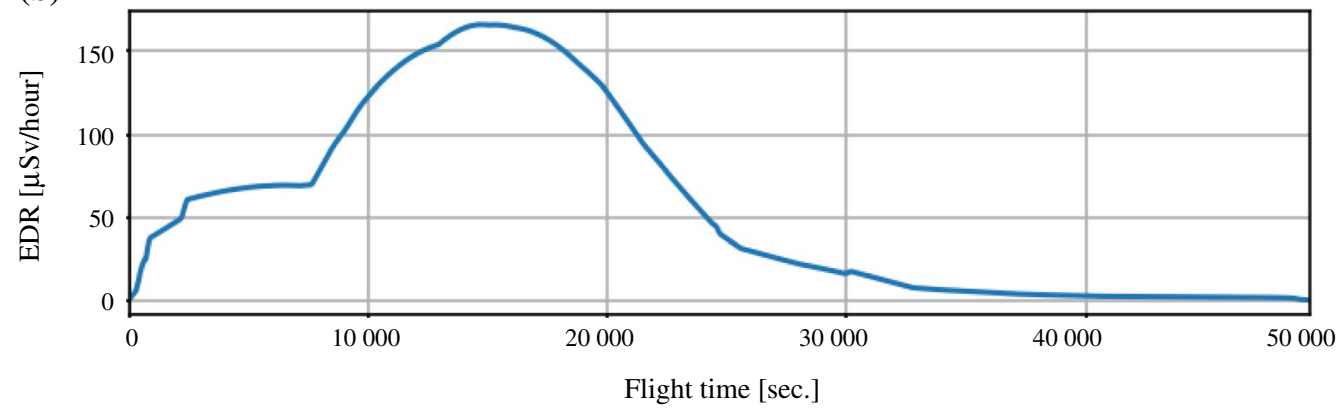

Fig. 3 a Flight altitudes and vertical profiles of estimated EDR and $\mathbf{b}$ estimated EDR along the reference flight path (Fig. 1) 


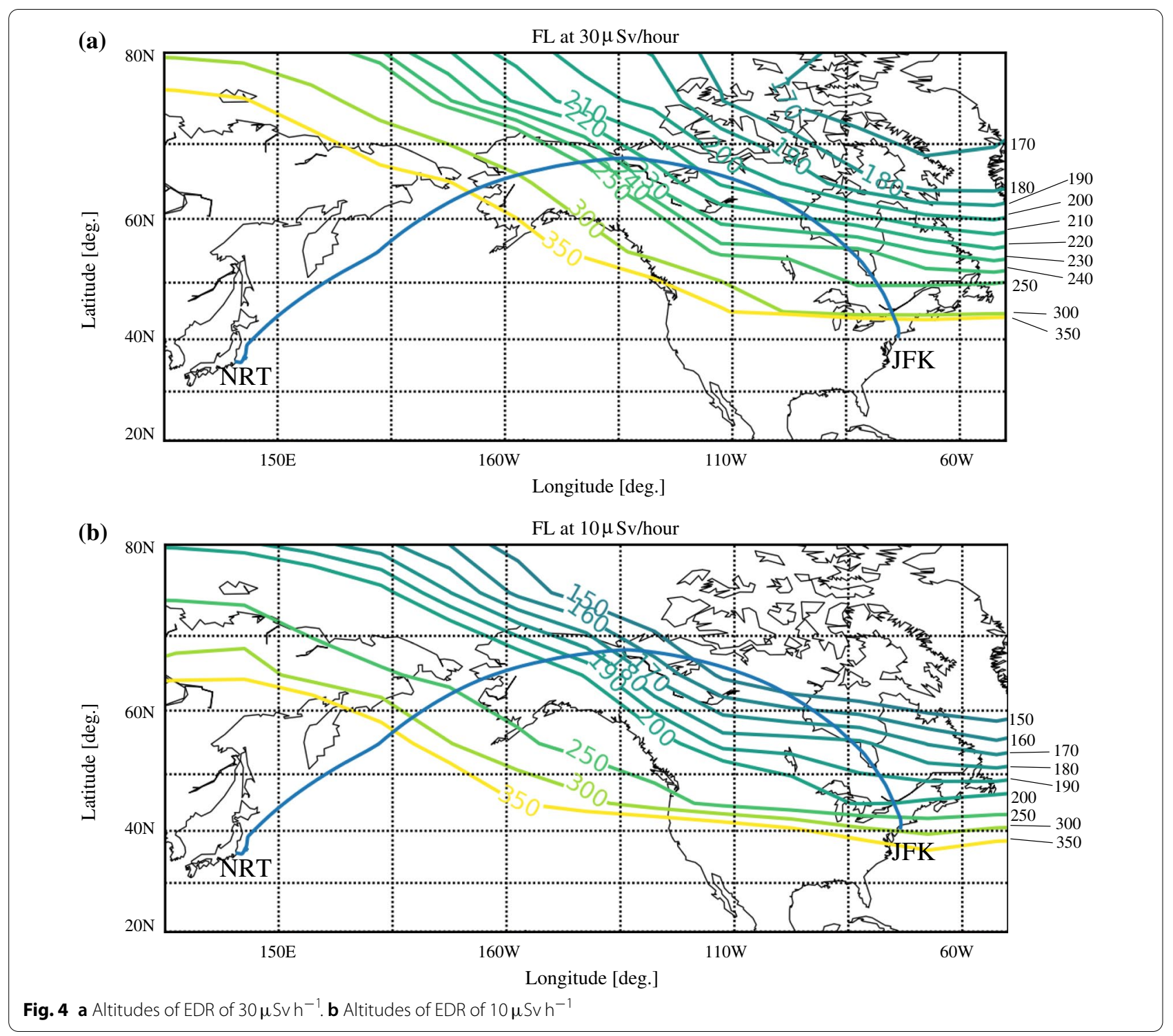

the GLE-69 class may cause economic impact as large as a few to several million JPY (some tens thousands USD) per flight.

These results should be considered as conservative limit cases. In most of cases, ARS effects at aircraft cruising altitudes are significant for a few hours (Sato et al. 2018). To evaluate the economic impacts in more probable cases, we have estimated them for a limited period of ARS events. As shown in Table 3, we set up additional two scenarios, Scenarios 6 and 7 , with constraints in the flight altitude in the first $3 \mathrm{~h}$ after departure, $\mathrm{FL}<200$ and $\mathrm{FL}<$ 140 , respectively. These scenarios represent a case where an ARS event has commenced before flight and operators reflect the ARS event in flight planning. The lateral flight paths were kept the same as the reference scenario
Table 1 Scenarios of flight trajectory calculation

\begin{tabular}{|c|c|c|c|c|c|}
\hline Scenario & $\begin{array}{l}1 \\
\text { (reference) }\end{array}$ & 2 & 3 & 4 & 5 \\
\hline $\begin{array}{l}\text { Threshold } \\
\text { in EDR } \\
{\left[\mu \mathrm{Svh}^{-1}\right]}\end{array}$ & - & 30 & 30 & 10 & 10 \\
\hline Constraints & - & $\mathrm{FL}<200$ & $\begin{array}{c}\mathrm{FL}<300 \\
\text { Lat. }< \\
44^{\circ} \mathrm{N}\end{array}$ & $\mathrm{FL}<140$ & $\begin{array}{c}\mathrm{FL}<250 \\
\text { Lat. }< \\
42^{\circ} \mathrm{N}\end{array}$ \\
\hline
\end{tabular}

(Scenario 1). These two scenarios can be regarded as the variations of Scenarios 2 and 4, respectively, with a limited ARS event period. After $3 \mathrm{~h}$ after departure, the aircraft are allowed to clime to the altitude of the reference scenarios. The altitude profiles of the Scenarios 6 


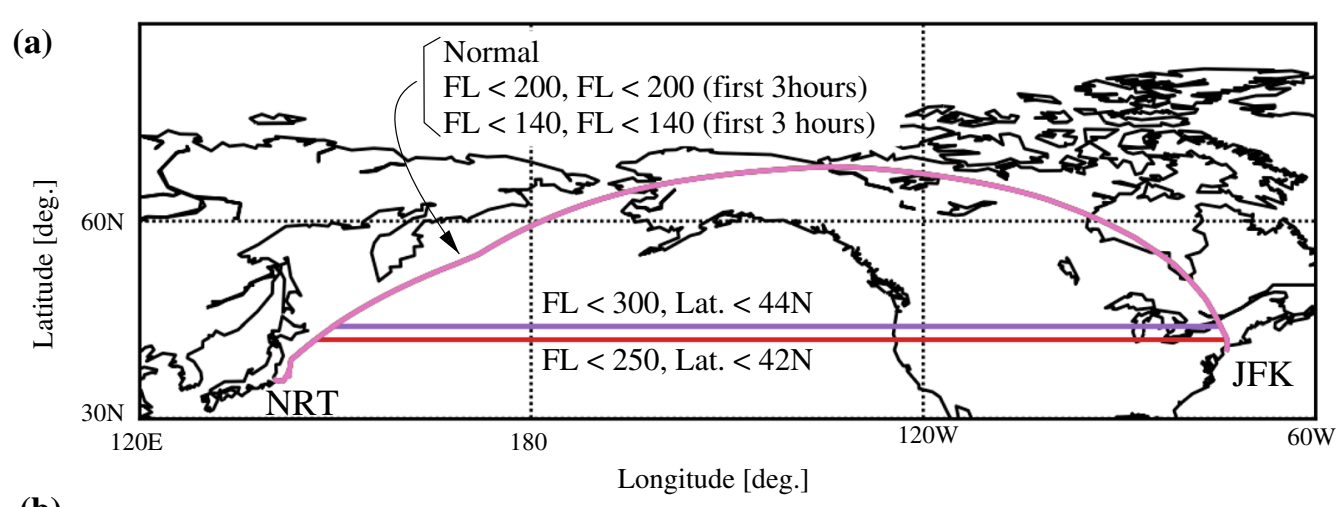

(b)

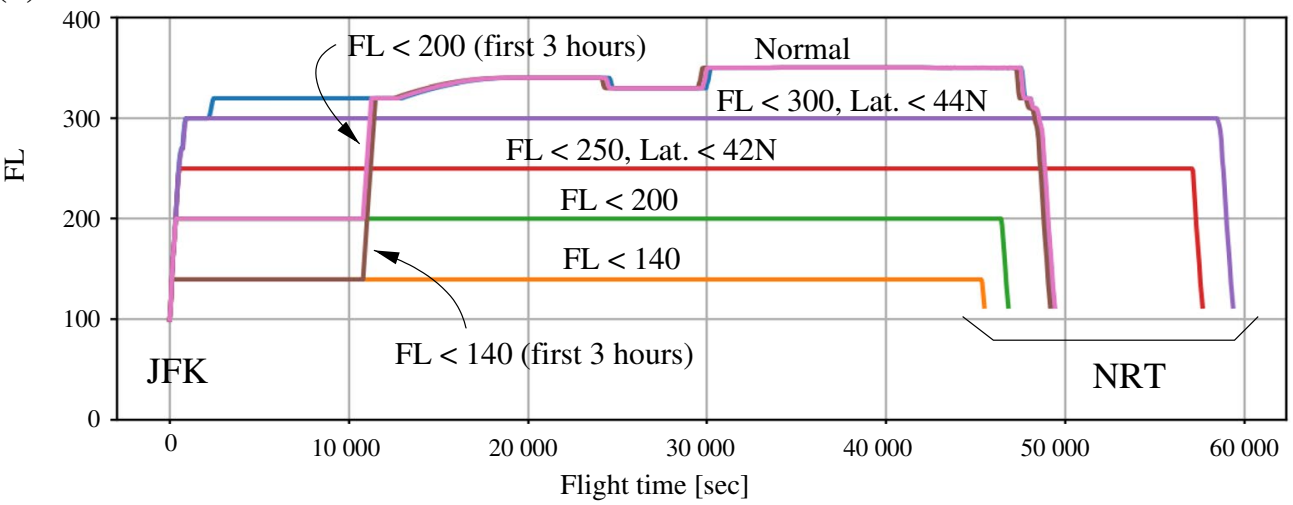

Fig. 5 a Lateral flight paths for each scenario. b Flight altitudes for each scenario

Table 2 Differences in the flight path length, flight time, and fuel consumption for JFK-NRT

\begin{tabular}{|c|c|c|c|c|c|}
\hline Scenario & 1 (reference) & 2 & 3 & 4 & 5 \\
\hline Flight path length $(\mathrm{km})$ & $11,334.6$ & $11,334.6$ & $12,798.2$ & $11,334.6$ & $12,911.6$ \\
\hline Increase (km) & - & 0.0 & 1463.6 & 0.0 & 1577.0 \\
\hline Increase (\%) & - & 0.0 & 12.9 & 0.0 & 13.9 \\
\hline Flight time (s) & 49,380 & 46,813 & 59,357 & 45,474 & 57,655 \\
\hline Increase (s) & - & -2567 & 9977 & -3906 & 8275 \\
\hline Increase (\%) & - & -5.2 & 20.2 & -7.9 & 16.8 \\
\hline Fuel consumption (kg) & 118,230 & 157,540 & 149,770 & 187,180 & 166,650 \\
\hline Increase (kg) & - & 39,310 & 31,540 & 68,950 & 48,420 \\
\hline Increase (\%) & - & 33.2 & 26.7 & 58.3 & 41.0 \\
\hline Fuel cost increase (million JPY) & - & 2.85 & 2.29 & 5.01 & 3.52 \\
\hline Threshold in EDR $\left(\mu \mathrm{Sv}^{-1}\right)$ & - & 30 & 30 & 10 & 10 \\
\hline Constraints & - & $F L<200$ & $\mathrm{FL}<300$, Lat. $<44^{\circ} \mathrm{N}$ & $\mathrm{FL}<140$ & $\mathrm{FL}<250$, Lat. $<42^{\circ} \mathrm{N}$ \\
\hline
\end{tabular}

The thresholds in EDR and constraints for each scenario (Table 1) are reproduced for reference

and 7 are shown in Fig. 5. The results of calculation for these additional scenarios are summarized in Table 3. Increases in the fuel consumption for Scenarios 6 and 7 were about 7.6 and 14 tons $(6.4$ and $12 \%$ of the reference scenario), corresponding to the fuel cost of 0.55 and 1.01 million JPY (or about 5.0 and 9.2 thousand USD), respectively. Comparing with Scenarios 2 and 4, the increase in the fuel consumption was reduced to about one-fifth, corresponding to the shortened flight time at lower altitudes. The total flight times for Scenarios 6 and 7 were increased from those of Scenarios 2 and 4, respectively, and they were almost the same as the reference scenario, although we assume that the aircraft maintains the original Mach speed profile. Because the air temperature is 
higher at lower altitudes, the corresponding air speed is higher. In our particular case, however, the head wind at lower altitude in the first $3 \mathrm{~h}$ was stronger than at higher altitudes and the ground speed was almost the same. As a result, the flight times were almost the same as the reference scenario. If the prediction of the ARS event duration would be available, operators could make a flight plan with such reduced constraints on the flight path to reduce the economic impacts.

This economic impact may be further reduced, if the space weather nowcast and forecast for the ARS associated with the SEP is utilized with an optimal flight trajectory generation algorithm (Wickramasinghe et al. 2014) to plan flights by considering the ARS impact prior to departure. As it can be seen in the comparison between scenarios with altitude constraint only (scenarios 2 and 4) and those with constraints on the altitude and latitude (scenarios 3 and 5), there would be the optimal combination of the altitude and latitude to minimize the economic impacts caused by the increased fuel consumption and increased flight time (delay of arrival). Setting more flexible constraints in the flight path to minimize the economic impacts by using the local EDR value derived from the global distribution as an additional constraint would be effective in providing optimal flight trajectories as well as estimating more realistic economic impacts, which is our next step. Furthermore, prediction of the progress of ARS associated with SEP events would be effective in reducing the economic impacts by adaptively modifying the flight path during the flight in a similar way as the Dynamic Airborne Reroute Procedure (DARP) which is deployed over the Pacific Ocean to avoid volcanic ashes or severe wind conditions. Although we assume that the EDR conditions at the peak of the ARS event is maintained all through the flight, normally the SEP flux would be decreased by time, and the EDR would also be decreased. When the ARS event ceases and the information is sent to the aircraft operator in a timely manner, the aircraft may be able to resume flying on a more efficient route and reduce the economic impact on the rest of the flight. If the progress of an ARS event is well predicted and it is informed to the aircraft operator, it would be possible to rearrange the flights in airspace to resume flying in more efficient routes.

Thus, the nowcasting and forecasting the ARS associated with the SEP events would be useful to reduce the economic impacts on the aircraft operation. It is even more important to integrate the space weather information and the optimal aircraft trajectory generation algorithm to develop a system to keep the traffic as efficient as possible by keeping safety with a minimal increased economic impacts.

In reality, the aircraft operation is a complex system, and the reaction to space weather events must be considered in broader sense. In the ARS events, communication means such as satellite communications (SATCOM) and high-frequency (HF) radio communication may also fail due to polar cap absorption, ionospheric storms, and scintillations. How to share information with aircraft in such conditions should also be considered for future. The flight routes of aircraft must be considered in the context of the air traffic management, because the flights cannot freely planned but must be determined by harmonizing the flow of air traffic. Aircraft in the air cannot change their flight routes without clearance by considering the air traffic condition around them. Therefore, our study is just a starting point to utilize the space weather information in civil aviation where various players are working collaboratively.

Table 3 Differences in the flight path length, flight time, and fuel consumption for JFK-NRT for ADR lasting in the first $3 \mathrm{~h}$ only

\begin{tabular}{llll}
\hline Scenario & $\mathbf{1}$ (reference) & $\mathbf{6}$ & $\mathbf{7}$ \\
\hline Constraints & - & $\mathrm{FL}<200$ in first 3 h & $\mathrm{FL}<140$ in first 3 h \\
Flight path length (km) & $11,334.6$ & $11,334.6$ & $11,334.6$ \\
Increase (km) & - & 0.0 & 0.0 \\
Increase (\%) & - & 0.0 & 0.0 \\
Flight time (s) & 49,380 & 49,407 & 49,196 \\
Increase (s) & - & -27 & -184 \\
Increase (\%) & - & -0.055 & -0.37 \\
Fuel consumption (kg) & 118,230 & 125,814 & 132,099 \\
Increase (kg) & - & 7584 & 13,869 \\
Increase (\%) & - & 6.4 & 11.7 \\
Fuel cost increase (million JPY) & - & 0.55 & 1.01 \\
\hline
\end{tabular}




\section{Conclusions}

In this study, we have estimated economic impacts of ARS associated with SEP events on aircraft operations. The global EDR distribution associated with the peak of ARS event on 20 January 2005 (GLE-69) is estimated by using WASVIES. For a flight route from JFK to NRT, four flight scenarios are set up to avoid hazard of ARS and are compared with a reference scenario which is the nominal case. In this study, thresholds on EDR below which the EDR should be are chosen as 30 and $10 \mu \mathrm{Svh}^{-1}$. For each EDR threshold, two strategies to constrain the flight path. One is to limit the flight altitude only, and the other is to limit the highest latitude with a relaxed constraint on altitude. Local solutions of flight path length, flight time, and fuel consumption are found for the five scenarios, including the reference and four scenarios to avoid radiation hazard, by using the trajectory generation algorithm with the BADA aircraft performance model. For a twin-engine, wide-body jet passenger aircraft, the flight time is reduced, but the fuel consumption is significantly increased by 39-69 tons (33-58\%) as compared to the reference scenario, when the constraint is set only on the aircraft altitude. When the constraints are set on the aircraft altitude and the latitude, flight time is increased by $2.2-2.8 \mathrm{~h}(17-20 \%)$. The increase in the fuel consumption is about $32-48$ tons (27-41\%) and is not as much as the scenarios with a constraint on the flight altitude only. If the ARS event duration is limited for $3 \mathrm{~h}$, the increase in the fuel consumption is about 7.6-14 tons (6.4-12\%).

This economic impact may be reduced, if the space weather nowcast for the ARS associated with the SEP and an optimal flight trajectory generation algorithm are used together. Setting more flexible constraints in the flight route and generating optimal flight trajectories with minimal economic impacts by fully utilizing the global EDR distribution would be effective, which is our next step. Furthermore, prediction of the progress of ARS associated with SEP events would be effective in reducing the economic impacts by adaptively modifying the flight path during the flight. Integration of the space weather information and the flight trajectory generation algorithm would be useful to keep the traffic as efficient as possible by keeping safety with a minimal increased economic impacts.

\section{Abbreviations \\ ARS: Atmospheric radiation storm; BADA: Base of Aircraft Data; EDR: Effective dose rate; FL: Flight level (in the unit of 100 feet); GLE: Ground level enhance- ment; ICRP: International Commission on Radiological Protection; ICAO: Inter- national Civil Aviation Organization; JPY: Japanese Yen; SEP: Solar energetic particle; USD: United States Dollar.}

\section{Acknowledgements}

The BADA model is developed by EUROCONTROL with data provided aircraft manufacturers.

\section{Authors' contributions}

SS designed this study, analyzed the equivalent dose rate on the flight path, and defined the criteria to constrain the flight path to avoid radiation. NW performed optimal aircraft path calculation and derived additional economic cost in aircraft operations. TS and DS provided the estimated global equivalent dose rate by SEP and galactic cosmic rays, and helped the interpretation of the results. All authors read and approved the final manuscript.

\section{Funding}

This study is supported by Grant-in-Aid for Scientific research on Priority Areas (15H05813) and Grant-in-Aid for Scientific Research (A) (15H02135) by Japan Society for the Promotion of Science (JSPS) and the Ministry of Education,

Culture, Sports, Science and Technology (MEXT) of Japan.

\section{Availability of data and materials}

The EDR data predicted by WASAVIES can be obtained at https://wasavies.nict. go.jp/index_e.html. The data on the calculated flight paths, flight times, and fuel consumption can be obtained upon request to the corresponding author (susaito@mpat.go.jp).

\section{Competing interests}

The authors declare that they have no competing interests.

\section{Author details}

${ }^{1}$ Electronic Navigation Research Institute, National Institute of Maritime, Port and Aviation Technology, 7-42-23 Jindaiji-Higashi, Chofu, Tokyo 182-0012, Japan. $^{2}$ Japan Atomic Energy Agency, 2-4 Shirakata, Tokai, Ibaraki 319-1195, Japan. ${ }^{3}$ National Institute of Information and Communications Technology, 4-2-1 Nukui-Kita, Koganei, Tokyo 184-8795, Japan.

Received: 6 August 2020 Accepted: 8 February 2021

Published online: 23 February 2021

\section{References}

ASTM (2020) D1655-20b, Standard Specification for Aviation Turbine Fuels, ASTM International, West Conshohocken, PA

Copeland K (2014) Cosmic ray particle fluences in the atmosphere resulting from primary cosmic ray heavy ions and their resulting effects on dose rates to aircraft occupants as calculated with MCNPX 2.7.0 Doctoral Thesis, Royal Military College of Canada, Kingston

Copeland K (2017) CARI-7A: Development and Validation. Radiation Protection Dosimetry 175:419-431. https://doi.org/10.1093/rpd/ncw369

International Civil Aviation Organization (ICAO) (2018a) Meteorological services for international air navigation, Annex 3 to the Convention on the International Civil Aviation, Amendment 78, ICAO, Montreal

International Civil Aviation Organization (ICAO) (2018b) Doc 10100, Manual on space weather information in support of international air navigation. ICAO, Montreal

International Commission on Radiological Protection (ICRP) (2007) The 2007 recommendations of the International Commission on Radiological Protection ICRP, Toronto

Kataoka R, Sato T, Kubo Y, Shiota D, Kuwabara T, Yashiro S, Yasuda H (2014) Radiation dose forecast of WASAVIES during ground- level enhancement Space Weather 12:380-386. https://doi.org/10.1002/2014sw001053

Lantos P, Fuller N (2003) History of the solar particle event radiation doses onboard aeroplanes using a semi-empirical model and Concorde measurements. Radiation Protection Dosimetry 104(3):199-210

Lantos P, Fuller N, Bottollier-Depois JF (2003) Methods for estimating radiation doses received by commercial aircrew. Aviation, Space, and Environmental Medicine 74:746-752

Latocha M, Beck P, Roller S (2009) AVIDOS_A software package for European accredited aviation dosimetry. Radiation Protection Dosimetry, 136:4, 286-290, doi10.1093/rpd/ncp126

Latocha M, Beck P, Butikofer R, Thommesen H (2015) AVIDOS 2.0_Aviation dosimetry service at ESA's space weather portal. paper presented at Space Weather Week, Boulder, USA, 13-17

Mattiä D, Schaefer M, Meier MM (2015) Economic impact and effectiveness of radiation protection measures in aviation during a ground 
level enhancement. J. Space Weather Space Climate 5:A17. https://doi. org/10.1051/swsc/2015014

Mekhaldi F, Musheler R, Adolphi F, Aldahan A, Beer J, McConnell JR, Possnert G, Sigl M, Svensson A, Synal H-A, Welten KC, WoodruffTE (2015) Multiradionuclide evidence for the solar origin of the cosmic-ray events of $774 / 5$ and 993/4. Nature Communications 6:8611. https://doi.org/10.1038/ ncomms 9611

Mertens CJ, Kress BT, Wiltberger M, Blattnig SR, Slaba TS, Solomon SC, Engel M (2010) Geomagnetic influence on aircraft radiation exposure during a solar energetic particle event in October 2003. Space Weather 8:S03006. https://doi.org/10.1029/2009sw000487

Mouillet V (2017) User manual for Base of Aircraft Data (BADA) Revision 3.14 EUROCONTROL Experimental Centre (EEC) Technical/Scientific Report No. 17/05/29-143

Poles M (2009) Base of Aircraft Data (BADA) aircraft performance modeling report. EUROCONTROL Experimental Centre (EEC) Technical/Scientific Report No. 2009-009

Sato T (2015) Analytical Model for Estimating Terrestrial Cosmic Ray Fluxes Nearly Anytime and Anywhere in the World: Extension of PARMA/EXPACS PLOS ONE 10:e0144679. https://doi.org/10.1371/journal.pone.0144679

Sato T, Kataoka R, Shiota D, Kubo Y, Ishii M, Yasuda H, Miyake S, Park I, Miyoshi Y (2018) Real time and automatic analysis program for WASAVIES: Warning System for Aviation Exposure to Solar Energetic Particles. Space Weather 16:924-936. https://doi.org/10.1029/2018SW001873
Wickramasinghe NK, Miyamoto Y, Harada A, Kozuka T, Shigetomi S, Miyazawa Y, Brown A, Fukuda Y (2014) Flight Trajectory Optimization for Operational Performance Analysis of Jet Passenger Aircraft. Trans. JSASS Aerospace Tech. Japan 12: APISAT-2013, a17-a25

Wickramasinghe NK, Brown MA, Fukushima S, Fukuda Y (2017a) OptimizationBased Performance Assessment on 4D Trajectory-Based Operations with Track Data. In: Air Traffic Management and Systems II. Selected Papers of the 4th ENRI International Workshop, Tokyo November 2015. Lecture Notes in Electrical Engineering, vol 420. Springer, Heidelberg, p 113

Wickramasinghe NK, Brown MA, Hirabayashi H, Nagaoka S (2017b) Feasibility Study on Constrained Optimal Trajectory Application in the Japanese Airspace. In: Proceedings of American Institute of Aeronautics and Astronautics Modeling and Simulation Technologies Conference, SciTech Forum 2017

FilightRadar24 (2020) FlightRadar24 AB, Sweden. https://www.flightradar24. com/commercial-services/data-services

WASAVIES (2020) National Institute of Information and Communications Technology, Japan. https://wasavies.nict.go.jp/index_e.html

\section{Publisher's Note}

Springer Nature remains neutral with regard to jurisdictional claims in published maps and institutional affiliations.

\section{Submit your manuscript to a SpringerOpen ${ }^{\circ}$ journal and benefit from:}

- Convenient online submission

- Rigorous peer review

- Open access: articles freely available online

- High visibility within the field

- Retaining the copyright to your article

Submit your next manuscript at $\boldsymbol{\nabla}$ springeropen.com 